\title{
Simulating LoRaWAN: on Importance of Inter Spreading Factor Interference and Collision Effect
}

\author{
$1^{\text {st }}$ Juho Markkula \\ Centre for Wireless Communications (CWC) \\ University of Oulu \\ Oulu, Finland \\ juho.markkula@oulu.fi
}

\author{
$2^{\text {nd }}$ Konstantin Mikhaylov \\ $C W C$ \\ University of Oulu \\ Oulu, Finland \\ konstantin.mikhaylov@oulu.fi
}

\author{
$3^{\text {rd }}$ Jussi Haapola \\ $C W C$ \\ University of Oulu \\ Oulu, Finland \\ jussi.haapola@oulu.fi
}

\begin{abstract}
The paper introduces a LoRaWAN simulation model implemented for the Riverbed Modeler (former OPNET modeler suite). First, the key components of the developed simulator solution are detailed. Then, the results of the simulator's validation for several test scenarios are presented. The developed simulator is used to investigate the effect of collision models on the results of LoRaWAN performance simulation. Specifically, the three models are studied: a baseline - implying loss off all colliding packets, an intra spreading factor (SF) with capture effect, and intra/inter SF with capture effect. The simulations verify that the results of the baseline model are in line with that for pure Aloha, while the two other demonstrate up to two-three fold higher delivery ratio. The obtained results illustrate the substantial impact of the collision model on the accuracy of simulations and motivate the need for further practical studies for the collision and interference mechanisms within a LoRaWAN network. Based on the results obtained through simulations, several drawbacks related to the use of strictly periodic traffic in LoRaWAN networks are noted.
\end{abstract}

\section{INTRODUCTION}

The number of physical devices connected to the Internet such as vehicles, home appliances, and other "things" has constantly been increasing over the past few years, thus forming the basis for the Internet of Things (IoT) [1]. The IoT ecosystem of today spans over the boundaries of countries and application domains, combining myriads of versatile devices, which differ in respect to their structures, capabilities, and available resources. Therefore, this is not surprising, that the landscape of the IoT-grade wireless communication technologies available today is excessively broad and diverse [2].

Low power wide area networks (LPWANs) are one of the most recent supplements to the IoT communication technology portfolio, which has already drawn substantial attention from academy and business alike. The LPWAN technologies address the need of having a cost-efficient communication solution for massive IoT with limited quality of service needs by reducing both the capital and operational expenditures for both IoT devices and the communication infrastructure. To achieve this, optimization of energy-efficiency, scalability, and coverage are especially important. Among the most widely used LPWA technologies, today are LoRaWAN, Sigfox, Narrowband-IoT (NB-IoT), and LTE-M [3].
According to the recent market analyses [3], LoRaWAN is today the most widely used LPWAN technology and is deployed today already in over 100 countries worldwide. The technology [4] enables deployment of both operator controlled wide area networks and private networks controlled by microoperators or individuals. The LoRaWAN networks feature a star-of-stars topology, having a network server (NS) in its core. One or multiple gateways (GWs) convey the data between the NS and end devices (EDs). Assuming the prevalence of uplink traffic above downlink, the LoRaWAN EDs feature Alohalike channel access mechanism with a random selection of a frequency channel to use. The downlink traffic is typically sent in one of the two receive windows (RWs), which are scheduled after an uplink transmission. The physical (PHY) layer of LoRaWAN is primarily based on a proprietary chirp spread spectrum modulation named LoRa. Depending on the distance between an ED and a GW, one of several orthogonal spreading factors (SFs) can be utilized. The higher SFs enable to increase the potential range of communication to several kilometers at the cost of increasing the on-air-time, which brings with it an increased energy consumption of the transmitting ED and a longer interference to other devices of the network.

The proliferation of LoRaWAN networks and the fast increase of the number of EDs deployed "in field" motivates the need for understanding the limits of LoRaWAN scalability. In this paper, we approach this problem by proposing a new, powerful LoRaWAN network simulation tool (compatible with version 1.1 specification [4]), which can be applied to verify technology improvements and to discover possible solutions to optimize the LoRaWAN network performance. The novelty of the simulation tool is mostly due to the more advanced collision and interference models incorporated in it, as discussed in the next section. To show the utility of the developed tool we first present the results of its validation, and then investigate how accounting of the different practical effects (specifically, capture effect and interference models) affect the obtained results. Our results, on one hand, show the substantial impact of accounting for these effects, and on the other hand, motivate further practical studies of the collision and interference mechanisms within a LoRaWAN network. 


\section{RELATED WORKS}

The number of devices a network can support is a key performance indicator for LPWANs. The research presented in [5] provides a brief analysis of the LoRaWAN communication technology, the potential throughput available to a single $\mathrm{ED}$, and the number of devices which can be served by a single GW. In [6], mathematical tools are applied to research the performance of a single-GW-LoRaWAN-network when inter-SF interference is considered as an independent link outage condition that decays the communication performance exponentially with increasing number of EDs. A single GW LoRaWAN scalability is investigated in [7] using a simulation model that applies the results of interference measurements between two EDs that transmit concurrently using the same SF. Particularly, the capture effect is researched, and the results show that even when concurrent transmissions occur, one packet may be received correctly under certain conditions.

Real-life experiments with LoRaWAN were reported in [8] and [9]. The results demonstrate that the transmissions using distinct SFs and even different frequency channels do negatively affect each other, but there is still a probability of receiving a packet correctly even if the interferer operates with the same SF. A table containing LoRaWAN co-channel rejection thresholds, i.e. the difference of the power levels for two concurrently received transmissions enabling reception of them, for different combinations of SFs is introduced in [10]. In [11], other co-channel rejection threshold tables are proposed based on Matlab simulations and actual measurement.

The work presented in [12] studied the impact of bidirectional traffic in LoRaWAN by extending the LoRaSim simulator to include downlink. The simulator does not consider inter-SF interference by assuming perfect orthogonality between SFs. In [13], an ns-3 network simulator module is developed, and its functionality is verified by comparing the simulation results with actual device measurements. The module is enhanced with carrier sense multiple access protocol to reduce the number of collisions and to overcome the duty cycle restrictions at the cost of increased energy consumption. However, many LoRaWAN functionalities are not simulated, such as channel hopping and downlink communications.

An ns-3 network simulator module in [14] is developed to study the performance of a LoRaWAN-based IoT network in a typical urban scenario and takes into account both inter and intra-SF interference. This is done by calculating the signal-to-interference-plus-noise ratio (SINR) and comparing the resulting value with the threshold table from [10]. The authors of [15] developed a LoRaWAN ns-3 module where the interference and the capture effect are modeled using BER curves obtained from complex baseband Matlab simulations. However, interfering transmissions using distinct SFs are equally considered as noise in SINR estimation. In its turn, the Riverbed Modeler network simulator solution of this paper combines the both in a two-stage procedure. First the threshold table presented in [10] is applied in a manner that is illustrated in [14] to model the inter and intra-SF interference. Next, if the threshold is exceed, the BER curves are utilized to perform the final decision of the packet acceptance based on the total SINR value.

\section{DeVEloped Simulator}

The tool in this work was developed for Riverbed Modeler [16] (former OPNET modeler suite) and represents a packet level, event-based simulation model. Each generated packet traverses sequentially through the blocks representing different layers of the communication stack (e.g., media access, PHY, physical channel), during which some headers are added or removed. The blocks are represented by $\mathrm{C}$ code and also include Riverbed Modeler specific functions. Riverbed Modeler was selected to be applied since the authors have successfully used it during their previous research [17]-[20], and it showed itself to be an extensive and accurate simulation tool. Also, in future, we consider combining the results of these studies to simulate a heterogeneous network composing multiple communication technologies. Meanwhile, in the following subsections, we discuss the most critical components of our developed simulator.

\section{A. Channel Model}

For modeling the path loss for our simulator we selected the well-known and often-used Hata Rural [21] model. The path-loss $\left(P L_{H R}\right)$ is

$$
\begin{aligned}
P L_{H R}[d B] & =P L_{H U}[d B]-4.78\left[\log \left(f_{c}\right)\right]^{2} \\
& +18.33 \log \left(f_{c}\right)-40.94,
\end{aligned}
$$

where $f_{c}$ is the center frequency in megahertz and $P L_{H U}$ is the Hata urban path-loss component. The $P L_{H U}$,

$$
\begin{aligned}
P L_{H U}[d B] & =69.55+26.16 \log \left(f_{c}\right)-13.82 \log \left(h_{c o}\right) \\
& -C_{H}+\left[44.9-6.55 \log \left(h_{c o}\right)\right] \log (d),
\end{aligned}
$$

where $h_{c o}$ is the antenna height of a GW in meters, $d$ is the distance between an ED and a GW in kilometers, and $C_{H}$ is the antenna height correction factor for a small or medium-sized city. $C_{H}$ can be calculated as

$$
C_{H}=\left[1.1 \log \left(f_{c}\right)-0.7\right] h_{e d}-\left[1.56 \log \left(f_{c}\right)-0.8\right],
$$

where $h_{e d}$ is the antenna height of an ED in meters. The signalto-noise ratio (SNR) $\left(S N R_{r x}\right)$ can be calculated as

$$
S N R_{r x}[d B]=P_{t x}[d B]+G_{t x}[d B]-P L_{H R}[d B]-N_{r x}[d B],
$$

where $P_{t x}$ is the transmission power, $G_{t x}$ is the transmitter antenna gain, and $N_{r x}$ is the received noise power as

$$
N_{r x}[d B]=N F+T_{b k g} B W_{r x} B,
$$

where $N F$ is the receiver noise figure $(6 \mathrm{~dB}), T_{b k g}$ is the background temperature $(293 \mathrm{~K}), B W_{r x}$ is the bandwidth per channel in herz, and $B$ is the Boltzmann constant. Note, that this channel model is used just as a reference and can be modified to a more appropriate one whenever needed (e.g., to better refract particular environment). 




Fig. 1. Bit error ratio as a function of $\mathrm{S}(\mathrm{I}) \mathrm{NR}$ for different spreading factors.

\section{B. Physical layer}

To model the operation of the PHY layer of LoRaWAN transceivers, we conducted a set of simulations in Matlab, from which the BER curves (depicted in Fig. 1) for various SNR for different SFs were obtained. The simulations were performed implying the additive white Gaussian noise (AWGN) channel and the Hamming code rate of 5/4. Average values of BER were calculated from the results of 10000 simulation iterations with SNR ranging from $-35 \mathrm{~dB}$ to $-5 \mathrm{~dB}$ with step size $\left(S_{\text {size }}\right)$ of $0.5 \mathrm{~dB}$.

Using the results of the Fig. 1 in a table form, the implemented simulator defines a BER value $\left(B E R_{S N R}\right)$ corresponding to the SF and the SNR or SINR of the received packet $\left(S(I) N R_{\text {rec }}\right)$. SNR value is applied if there are no concurrent transmissions on the same frequency channel, otherwise a collision is detected, and SINR is calculated and handled as this is described in Subsection III-C. First, the simulator fetches two BER values $\left(B E R_{\min }\right.$ and $\left.B E R_{\max }\right)$ from the table corresponding to the SNR values that are above $\left(S N R_{\max }\right)$ and below the $S(I) N R_{\text {rec }}$ value. Next, $B E R_{S N R}$ are calculated as

$$
\begin{aligned}
B E R_{S N R}=B E R_{\min }+ & \left(B E R_{\max }-B E R_{\min }\right) \\
& \times \frac{\left(S N R_{\max }[d B]-S(I) N R_{\text {rec }}[d B]\right)}{S_{\text {size }}} .
\end{aligned}
$$

Finally, the probability of the packet being correctly received, denoted $P_{\text {rec }}$, is calculated as

$$
P_{\text {rec }}=\left[1-B E R_{S N R}\right]^{n_{b}},
$$

where $n_{b}$ is the number of payload bits in a packet.

\section{Packet collision modes}

An uplink packet collision is registered by the GW if there exist at least two simultaneous transmissions on the same frequency channel. To study how accounting of the different effects change the simulation results, we introduced and implemented the three collision models:

- $\mathbf{B}(\mathbf{P})$ - baseline (pessimistic) - simultaneous transmissions cause discarding of all concurrent packets.
- IC - intra-SF collisions with capture effect - only the concurrent transmissions with the same SF may cause packet failures, but the transmissions with different SFs do not affect each other. Capture effect, permitting the GW to receive the packet with the highest SINR for each $\mathrm{SF}$ and channel, is enabled.

- IIC - transmissions with the same as well as with the other SFs may cause packet failures. The capture effect for the same SF is enabled.

The two former models are currently extensively used by the existing simulation tools (c.f. Section II). To determine whether a packet in modes IC and IIC is received correctly or not, we implement a two-stage procedure. First, we estimate the co-channel S(I)NR caused by the other simultaneous transmissions with each distinct $\mathrm{SF}\left(S F_{x}\right)$ separately as

$$
\operatorname{SINR}_{r x\left(S F_{x}\right)}[d B]=10 \log \frac{S N R_{r x \_r e a l}}{\left(1+S N R_{\text {intf_sum_real }\left(S F_{x}\right)}\right)},
$$

where $S N R_{r x_{-} r e a l}$ is the SNR value of the desired transmission and $S N R_{\text {intf_sum_real }\left(S F_{x}\right)}$ is the sum of the equalized SNR values of all interfering transmissions with the same $\mathrm{SF}\left(S F_{x}\right)$. During the equalization, the SNR of an interferer signal is multiplied with the overlapping ratio (i.e., the overlapping time of the desired and the interferer signal divided by the duration of the desired signal). In IC model the $\operatorname{SINR}_{r x}\left(S F_{x}\right)$ is calculated over the packets transmitted on the same channel only with the same SF as the desired transmission. For IIC, the $S_{I N R_{r x}\left(S F_{x}\right)}$ is calculated for each possible SF pair of the desired and interfering transmissions. Then $\operatorname{SINR}_{r x\left(S F_{x}\right)}$ are compared against the thresholds given in Table I [14] (note, that this table may easily be replaced with another one if needed). If the SINR does not exceed a threshold (note that a single threshold is checked in IC model; in IIC model the thresholds for all possible SF combinations are checked), the packet is considered to be erroneous. If the $\operatorname{SINR}_{r x\left(S F_{x}\right)}$ values are above the thresholds, the BER is determined based on the SINR, and the packet reception probability is calculated as described in Subsection III-B. Note, the cumulative SINR is utilized for determining BER in IIC model, which is given by

$$
\operatorname{SINR}_{r x(\text { total })}[d B]=10 \log \frac{S N R_{r x_{-} \text {real }}}{\left(1+\sum_{S F=7}^{12} S N R_{\text {int__sum_real }(S F)}\right)} .
$$

\section{Media access and network layers}

The media access layer was implemented as prescribed by the LoRaWAN specification for EDs of class A. Both uplink and downlink data transfers were enabled. In the current version of the model, we limited the network to a single GW. Due to this reason, the functionalities of the NS and the GW in the developed simulation model were combined within a single device. The duty-cycle restriction mechanism, preventing transmission in the same frequency channel for a particular amount of time after it has been used, has been implemented for both EDs and the GW. 
TABLE I

COCHANNEL SINR THRESHOLDS (DB) WITH ALL COMBINATIONS OF SFS FOR A DESIRED SIGNAL WHEN AN INTERFERER SIGNAL EXISTS (BASED ON [14]).

\begin{tabular}{c||c|c|c|c|c|c}
\hline \multicolumn{1}{c||}{$\begin{array}{c}\text { Desired } \\
\text { SF }\end{array}$} & \multicolumn{7}{c}{ Interferer SF } \\
\cline { 2 - 7 } & 7 & 8 & 9 & 10 & 11 & 12 \\
\hline $\mathbf{7}$ & 6 & -16 & -18 & -19 & -19 & -20 \\
\hline $\mathbf{8}$ & -24 & 6 & -20 & -22 & -22 & -22 \\
\hline $\mathbf{9}$ & -27 & -27 & 6 & -23 & -25 & -25 \\
\hline $\mathbf{1 0}$ & -30 & -30 & -30 & 6 & -26 & -28 \\
\hline $\mathbf{1 1}$ & -33 & -33 & -33 & -33 & 6 & -29 \\
\hline $\mathbf{1 2}$ & -36 & -36 & -36 & -36 & -36 & 6 \\
\hline
\end{tabular}

TABLE II

KEY PARAMETERS FOR THE SIMULATION SCENARIOS

\begin{tabular}{l|c}
\hline Parameter & Value \\
\hline Channel band width & $125 \mathrm{kHz}$ \\
\hline Base frequency & $868.1-868.9$ and $869.525 \mathrm{MHz}$ (CH 1-6) \\
\hline Transmission power & $0 \mathrm{dBi}$ \\
\hline Tx antenna gain & $3 \mathrm{~m}$ (end device), 24 m (gateway) \\
\hline Antenna height & ALOHA, random frequency channel \\
\hline MAC & Disabled \\
\hline Retransmissions & Disabled \\
\hline ADR and power control & 1s \\
\hline RECEIVE_DELAY1 & same as uplink \\
\hline RW1 SF & same as uplink \\
\hline RW1 channel & SF12 \\
\hline RECEIVE_DELAY2 & 869.525 MHz (CH 6) \\
\hline RW2 SF & by personalization \\
\hline RW2 channel & \\
\hline Activation: & \\
\hline
\end{tabular}

\section{Simulation Setup AND Scenario}

\section{A. Common Parameters}

The key parameters for the simulation scenarios are summarized in Table II. The EDs in our simulations were operating as class A LoRaWAN EDs with statically assigned SFs and transmit power. We also assume that a GW can receive packets on all the frequency channels $(\mathrm{CHs})$ and using all possible SFs simultaneously. The uplink packets are generated periodically and sent with no retransmissions. If downlink transmissions are present, the GW attempts to convey them in RW1 and uses RW2 if the transmission in RW1 is not possible due to the duty cycle restrictions, and refrains from transmitting a downlink packet if both RW1 and RW2 are blocked due to the duty cycle restrictions. The packet durations and the maximum capacity of a single channel (calculated as packet size in bits divided by packet on-air time) for distinct LoRaWAN SFs are presented in Table III. We assume that a packet is composed of 8-bit application (APP) layer payload, 64 bits MAC layer overhead, and 40 bits PHY overhead, resulting in the total packet size of $n_{b}=112$ bits.

\section{B. Simulation Setup}

To confirm the correct operation of the developed simulator and to investigate the effects of the different collision models we have investigated six test cases in this paper. The key simulation parameters for the cases are listed in Table IV.
TABLE III

MAXIMUM CHANNEL CAPACITY AND PACKET DURATION USING DISTINCT LORAWAN SFs.

\begin{tabular}{c|c|c}
\hline SF & Maximum capacity (kb/s) & Packet duration (ms) \\
\hline 7 & 2.417 & 46.3 \\
\hline 8 & 1.359 & 82.4 \\
\hline 9 & 0.679 & 164.9 \\
\hline 10 & 0.388 & 288.8 \\
\hline 11 & 0.170 & 659.5 \\
\hline 12 & 0.097 & 1155.1 \\
\hline
\end{tabular}

Note, the value of 1 erlang (E) refers to the full utilization of the network capacity within a single channel. The packet generation interval of a single ED can be calculated as a packet duration $\left(P_{d u r}\right)$ (Table III) multiplied by the total number of EDs (e.g., 300) and divided by the total ED traffic in E. DC denotes the maximum percentage of time a device (ED or GW) can spend transmitting in a channel. After a transmission, a device has to wait a time offset $\left(100 P_{d u r} / \mathrm{DC}-P_{d u r}\right)$ prior to a next transmission attempt. If a packet is generated during this time offset, the packet is discarded because no buffering is performed to wait until the channel becomes available again.

Throughout the simulations, all EDs start generating their traffic at a random time instant. In the test cases involving just a single ED, the interpacket intervals are constant. In the case of multiple EDs (i.e., the "network" cases) the Poisson distribution with particular mean packet generation interval is applied. In the two following sections, the setups for single ED and network simulation parameters are detailed further.

1) Single ED cases: The simulations including only one ED are primarily intended to check the correctness of the simulator's operation and the validity of the obtained results. For all these sub-cases a single ED was sending uplink traffic to a single GW. For each value of the parameters (i.e., each point on the charts), 50 hours of the system's operation were simulated. In total, four different sub-cases were simulated:

- S1 - the SF and the distance between the ED and the GW were varied in order to investigate the effect of the distance on the packet delivery ratio (PDR) under no interferences.

- S2 - the generated data traffic and DC limitations were varied to characterize their effect on the fundamental performance limits.

- S3 - the number of frequency channels was varied to explore their effect on the performance.

- S4 - the downlink communication from GW to EDs was enabled to study its effect on system performance.

For cases S2-S4 the ED was placed close enough to the $\mathrm{GW}$ to enable practically error-free communication.

2) Network cases: After confirming the correct operation of the simulator for single ED cases, the network was scaled up to include $300 \mathrm{EDs}$. The cumulative traffic in the network is generated as $10 \%, 20 \%, \ldots, 100 \%$ of the maximum capacity (i.e., $0.1,0.2, \ldots, 1 \mathrm{E}$ ). The $\mathrm{GW}$ was placed in the center of the simulated area, while the EDs were distributed uniformly within a $13 \mathrm{~km}$ zone, thus allowing for close to $100 \%$ 
TABLE IV

PARAMETERS FOR SIX SIMULATED CASES.

\begin{tabular}{|c|c|c|c|c|c|}
\hline Single end device cases: & $\begin{array}{c}\text { End device } \\
\text { traffic }\end{array}$ & $\begin{array}{l}\begin{array}{c}\text { Distance to the } \\
\text { gateway }\end{array} \\
\end{array}$ & $\begin{array}{c}\text { Spreading } \\
\text { factors }\end{array}$ & Duty cycle $[\%]$ & $\begin{array}{c}\begin{array}{c}\text { Frequency channel } \\
\text { identifiers }\end{array} \\
\end{array}$ \\
\hline S1. PDR vs distance & generation interval: $2 \mathrm{~s}$ & $10,11, \ldots, 50 \mathrm{~km}$ & $7,8, \ldots, 12$ & 100 & \multirow{2}{*}{1} \\
\hline S2. Duty cycle limitations & \multirow{3}{*}{$\begin{array}{l}0.001,0.0033,0.005 \\
0.01,0.05,0.1,0.5 \mathrm{E} \\
0.001,0.0033,0.005 \\
0.01,0.02, \ldots, 0.05 \mathrm{E} \\
\end{array}$} & \multirow{3}{*}{$10 \mathrm{~km}$} & \multirow{2}{*}{7} & $0.333,1,10,100$ & \\
\hline S3. Channel hopping & & & & 1 & $1,2, \ldots, 5(2-5 \mathrm{CHs})$ \\
\hline S4. Downlink transmissions & & & $\begin{array}{l}10 \text { (RW1) } \\
12 \text { (RW2) }\end{array}$ & $\begin{array}{l}\text { (RW1, RW2): } \\
(1,1),(1,10)\end{array}$ & 1 (RW1), 6 (RW2) \\
\hline Network cases: (300 EDs) & & & & & \\
\hline $\begin{array}{l}\text { N1. Single SF } \\
\text { N2. Multiple SF }\end{array}$ & $0.1,0.2, \ldots, 1 \mathrm{E}$ & $\begin{array}{c}\text { Circular topology, } \\
\text { random radius: } 0-13 \mathrm{~km}\end{array}$ & $\begin{array}{c}7 \\
7,8, \ldots, 12\end{array}$ & 1 & 1 \\
\hline
\end{tabular}

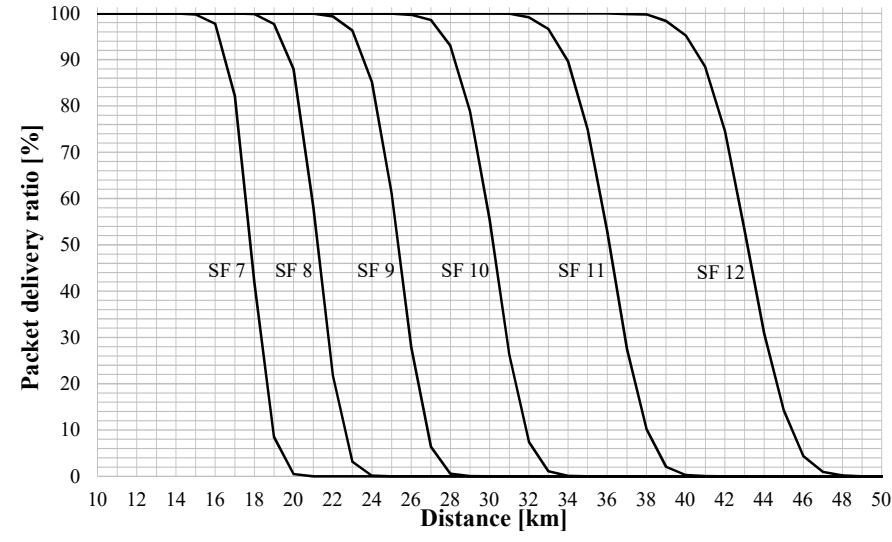

Fig. 2. Rate of correctly received packets as a function of transmission distance for distinct SFs.

PDR when no collisions are detected. Two sub-cases were investigated:

- N1 - all EDs operate with SF7, traffic varied from 0.1 to $1 \mathrm{E}, \mathrm{B}(\mathrm{P})$ and $\mathrm{IC}$ collision models are used. One hundred instances of two-hour-long simulations were run for each set of parameters, and the results were statistically processed.

- N2 - the SF7-SF12 were assigned to EDs in a random manner (50 EDs per each SF), traffic varied from 0.1 to $1 \mathrm{E}$, all three collision models investigated. One hundred instances of five-hour duration simulations were run for each parameter set.

\section{Simulation Results}

1) Single ED: The selected results of $\mathrm{S} 1$ case are presented in Fig. 2, depicting the percent of the correctly received packets as a function of transmission distance. When operating with SF7, all the packets were correctly received with a maximum distance of $13 \mathrm{~km}$. The corresponding distances for SF8-SF12 were 17, 20, 24, 29, and $35 \mathrm{~km}$, respectively. For $99 \%$ PDR the distances are 15, 18, 22, 26, 32, and 38 $\mathrm{km}$ for SF7-SF12, respectively. These distances may seem somewhat overoptimistic when compared to the results of empirical measurements (e.g., [22]). Nonetheless, the use of a well-established model enables for easier comparison of the obtained results and that in the other works.

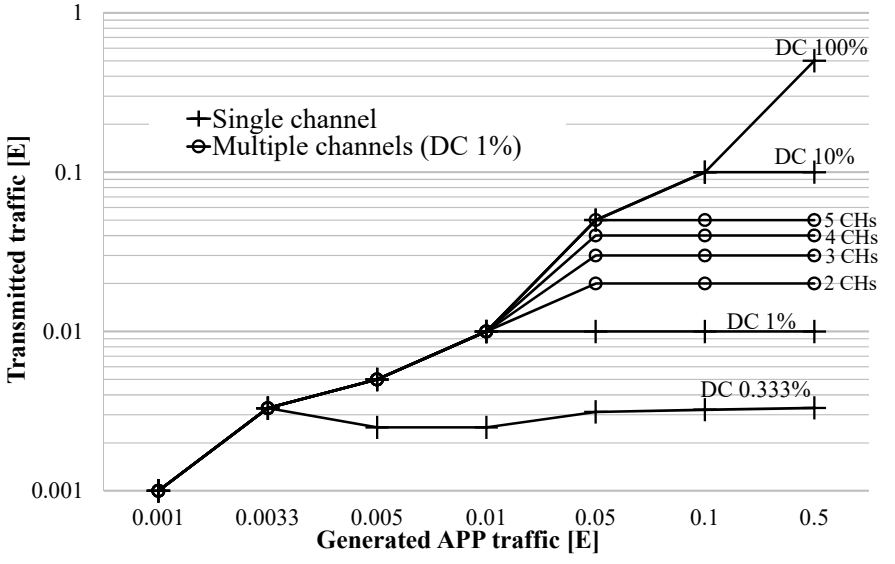

Fig. 3. Transmitted traffic as a function of generated traffic with distinct DC values and different number of $\mathrm{CHs}$ for channel hopping.

The results of S2 and S3 simulations are combined and presented in Fig. 3. The chart illustrates the transmitted traffic as a function of generated application layer traffic with distinct DC values and the different number of channels. Specifically, when $\mathrm{x}$ and $\mathrm{y}$ values are identical, all the generated APP traffic is successfully transmitted. The DC restrictions may prevent transmission of some packets, while the increase of available channels linearly increases the maximum transmitted traffic. Note, that all the transmitted packets were correctly received.

An interesting phenomenon can be noted for DC of $0.333 \%$ and the generated traffic of $0.005,0.01$ and $0.05 \mathrm{E}$; the transmitted traffic is below that generated for $0.0033 \mathrm{E}$. The reasons of such behavior are the strict periodicity of the generated traffic, lack of buffering and the duty cycle back-off mechanisms used in LoRaWAN. In the case, if by the moment next packet is generated, and the back-off timer has not expired, this packet is dropped. The next check of the channel is done once a new packet is generated, which occurs after another period duration. To give an example, $0.005 \mathrm{E}$ traffic corresponds to the packet generation period of approximately $9.3 \mathrm{~s}$. With DC $0.333 \%$ a period between SF7 packets cannot exceed $13.9 \mathrm{~s}$. Thus, in practice, only one of each two packets is transmitted.

The key results of S4 simulations are illustrated in Fig. 4. In this set up the GW was configured to transmit in a downlink in response to each uplink transmission. The chart illustrates 


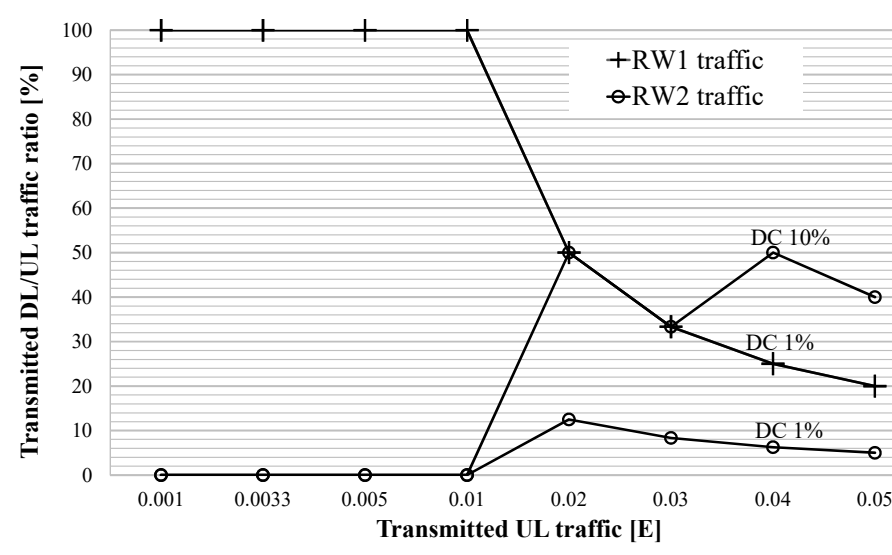

Fig. 4. Transmitted DL/UL traffic ratio in percentages using RW1 and RW2 as a function of transmitted UL traffic.

the ratio between the uplink and downlink packets, as well as the distribution of the downlink packets between RW1 and RW2. As one can see, under low channel load, all the uplink frames were acknowledged in RW1. With the increase of the uplink traffic, GW had to also use the RW2 for its downlink in the case when due to DC limitations it was not able to use RW1. Finally, once the amount of UL traffic exceeded 0.01 $\mathrm{E}$ under $1 \%$ DC limit, or $0.02 \mathrm{E}$ under $10 \% \mathrm{DC}$ limit the $\mathrm{GW}$ was not able to respond to all the packets. Interestingly, with the uplink traffic of $0.05 \mathrm{E}, 25 \%$ (20\% in RW1, 5\% in RW2) of the uplink packets were acknowledged when the both RWs had DC limitation of $1 \%$, and $60 \%$ (20\% in RW1, $40 \%$ in RW2) were acknowledged when the DC limitation of RW2 was increased to $10 \%$. Also, one can see that for $0.03 \mathrm{E}$ traffic the number of downlink packets in RW2 with $10 \%$ DC is below that for 0.02 or $0.04 \mathrm{E}$. This is also caused by the combined effect of the periodicity of the traffic and the DC restrictions.

2) Network: The results of N1 and N2 cases are combined in Fig. 5. The chart illustrates the throughput $(S)$ as a function of transmitted traffic $(G)$. In addition to the results of the simulations, the theoretical performance for pure Aloha $\left(S=G e^{-2 G}\right)$ [23] is also plotted (the lowest series). Note that in $\mathrm{N} 1$ all the EDs operate with SF7, having on-air time for a single packet equal to $46.3 \mathrm{~ms}$. For N2 the average packet duration is approximately $399.5 \mathrm{~ms}$, since all the SFs $7,8, \ldots, 12$ are utilized equally. Therefore, the number of the packets transmitted in N1 case exceeds that for N2 for the same generated traffic value (i.e., 21.6 against 7.6 packets per second correspond to $1 \mathrm{E}$ ).

Analyzing the results of $\mathrm{N} 1$, one can see that the results for $\mathrm{B}(\mathrm{P})$ match closely with the theoretical results for pure Aloha (the difference is about $0.115 \%$ ). Accounting for intraSF collisions and the capture effect (IC model) resulted in more than doubling the throughput, which is primarily due to the capture effect enabling a packet to be received even under the presence of interference.

Comparing the results of $\mathrm{N} 2$ case with pure Aloha, one can see that in $\mathrm{N} 2$ case the throughput is even higher. The

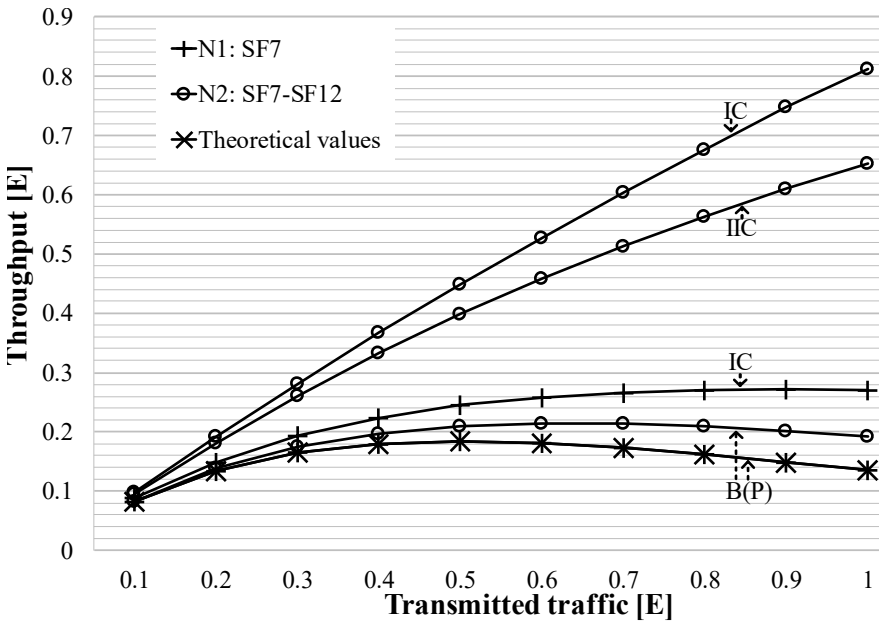

Fig. 5. Throughput as a function of transmitted traffic (N1 and N2).

difference increases, from 0.9 to $41.6 \%$, when the transmitted traffic amount is increased from 0.1 to $1 \mathrm{E}$. The phenomenon occurs because when increasing the traffic the packets with shorter duration sent with lower SF values are more likely to be correctly received without packet collisions than the transmissions with higher SF values.

Analyzing the results for the different collision modes, one can see that for $\mathrm{N} 2$ case the IC model increases the maximum throughput by 18.4 to $323.7 \%$ compared to the baseline (i.e., $\mathrm{B}(\mathrm{P})$ ) scenario. Accounting for inter-SF interference (IIC model), the performance is further decreased by 3.1 to $83.7 \%$ from IC model (making it 15.3 to $240.3 \%$ higher than $\mathrm{B}(\mathrm{P})$ model).

\section{CONCLUSION}

In the paper, a LoRaWAN LPWAN simulation model for the Riverbed modeler network simulator is presented. We detailed the design of the key components of the developed simulator and validated the main functionalities of the developed solution, which include: collision and capture effect, uplink and downlink transmissions, pure Aloha media access, detailed physical layer propagation model, support for distinct spreading factors, channel hopping, and duty cycle limitations for frequency channels. The correct operation of the simulator is verified with single end device cases and by comparing network simulation results with theoretical values for pure Aloha.

To investigate how strongly does the collision model affect the results of LoRaWAN network simulation, we conducted a set of simulations with three different models. The cumulative throughput obtained with collision models accounting for inter and intra-SF interference and the presence of capture effect was found to be able to substantially exceed that obtained with the baseline model, which implies that every collision causes loss of all colliding packets. Specifically, when using SF7, the difference between throughput values was from 7.1 to $100.1 \%$. With multiple SF (SF7-SF12), the difference was from 18.4 to $323.7 \%$ when accounting for the intra-SF interference 
and from 15.3 to $240.3 \%$ when accounting for the inter-SF interference.

The results of this work, on one hand, show that the developed tool is ready to be used for the variety of studies related to network behavior of LoRaWAN devices. At the same time, they illustratively show the importance of developing and detailing further the collision models for LoRaWAN, which are mostly missing as of today. Among other findings of this work can be noted:

- The negative effect of the strictly periodic traffic, when combined with duty cycle limitations introduced for LoRaWAN, on the channel utilization. Further optimizations (e.g., of the packet generation intervals) may enable addressing this issue.

- In order to increase the utilization of the downlink under strictly periodic uplink traffic, the selection of RW needs to be optimized.

The future work will contain the integration of the current model with the LTE network to form a hybrid LoRaWANLTE network simulator similarly as previously presented in [18]. The aim is to research the suitability of the hybrid solution for smart grid communications. The previous research [24] suggests that the hybrid LoRaWAN-LTE network may be suitable for automatic meter reading traffic and this simulation will validate this hypothesis. Furthermore, the modifications for LoRaWAN to improve the reliability and the scalability will be considered.

\section{ACKNOWLEDGMENT}

This research has been partially financially supported by Academy of Finland 6Genesis Flagship (grant 318927) and partially funded by the ITEA3 project APPSTACLE (15017).

\section{REFERENCES}

[1] A. Al-Fuqaha, M. Guizani, M. Mohammadi, M. Aledhari, and M Ayyash, "Internet of things: a survey on enabling technologies, protocols, and applications," IEEE Commun. Surv. \& Tutor., vol. 17, pp. $2347-$ 2376, Fourthquarter 2015

[2] S. Andreev et al., "Understanding the IoT connectivity landscape: a contemporary M2M radio technology roadmap,' IEEE Commun. Mag., vol. 53, pp. 32-40, Sept. 2015

[3] A. Weissberger, "LoRaWAN and Sigfox lead LPWANs; interoperability via compression," [Online]. Available: http://techblog.comsoc.org/2017/10/25/lora-wan-and-sigfox-leadlpwans-interoperability-via-compression/

[4] N. Sornin and A. Yegin (Eds.), "LoRaWAN 1.1 Specification," LoRa Alliance, Oct. 2017.

[5] K. Mikhaylov, J. Petäjäjärvi, and T Hänninen, "Analysis of capacity and scalability of the LoRa low power wide area network technology," Eur. Wireless 2016; 22th Eur. Wireless Conf., pp.1-6, June 2016.

[6] O. Georgiou and U Raza, "Low power wide area network analysis: can loRa scale?," IEEE Wireless Commun. Lett., vol. 6, pp.162-165, Apr. 2017.

[7] J. Haxhibeqiri, F. Van den Abeele, I. Moerman, and J. Hoebeke, "LoRa scalability: a simulation model based on interference measurements," MDPI Sensors, vol. 17, p. 1193, May 2017.

[8] K. Mikhaylov, J. Petäjäjärvi, and J. Janhunen, "On LoRaWAN scalability: empirical evaluation of susceptibility to inter-network interference," In Proc. Eur. Conf. Netw. Commun., pp. 1- 6, June 2017.

[9] K. Mikhaylov, J. Petäjäjärvi, and A. Pouttu, "Effect of Downlink Traffic on Performance of LoRaWAN LPWA Networks: Empirical Study," In Proc. Personal Indoor Mobile Wireless Commun. Conf., pp. 1- 6, September 2018.
[10] C. Goursaud and J.-M. Gorce, "Dedicated networks for IoT : PHY / MAC state of the art and challenges," EAI endorsed trans. on Internet of Things, Oct. 2015.

[11] D. Croce, M. Gucciardo, S. Mangione, G. Santaromita, and I. Tinnirello, "Impact of LoRa imperfect orthogonality: analysis of link-level performance," IEEE Commun. Lett., vol. 22, pp.796-799, Apr. 2018.

[12] A. Pop, U. Raza, P. Kulkarni, and M. Sooriyabandara, "Does bidirectional traffic do more harm than good in LoRaWAN based LPWA networks?," IEEE Glob. Commun. Conf., pp. 1-6, Dec. 2017.

[13] T.-H. To and A. Duda, "Simulation of LoRa in NS-3: improving LoRa performance with CSMA," IEEE Inter. Conf. on Commun. (ICC), pp. 1-7, May 2018.

[14] D. Magrin, M. Centenaro, and L. Vangelista, "Performance evaluation of LoRa networks in a smart city scenario," IEEE Inter. Conf. on Commun. (ICC), July 2017.

[15] F.Van den Abeele, J. Haxhibeqiri, I. Moerman, and J. Hoebeke, "Scalability analysis of large-scale LoRaWAN networks in ns-3," IEEE Internet of Things Jour., vol. 4, pp.2186-2198, Oct. 2017.

[16] Riverbed (2018, Oct.) Web page. [Online]. Available: http://www.riverbed.com

[17] J. Markkula and J. Haapola, "Impact of smart grid traffic peak loads on shared LTE network performance," IEEE Inter. Conf. on Commun (ICC), pp. 4046 - 4051, June 2013.

[18] J. Markkula and J. Haapola, "LTE and hybrid sensor-LTE network performances in smart grid demand response scenarios," IEEE Inter. Conf. on Smart Grid Commun. (SmartGridComm), pp. 187 - 192, Oct. 2013.

[19] J. Markkula and J. Haapola, "Ad hoc LTE method for resilient smart grid communications," Wireless Pers. Commun., vol. 98, pp. 3355-3375, Oct. 2017.

[20] J. Markkula and J. Haapola, "Impact of shared LTE network high typical traffic loads on smart grid demand response schemes," 3rd Inter. Conf. on Smart and Sustainable Technologies (SpliTech), pp. 1 - 6, June 2018.

[21] A.F. Molisch, Wireless Communications, 2nd ed. Chichester, UK: John Wiley \& Sons Ltd, 2011, Appendix 7.A.

[22] J. Petäjäjärvi, M. Pettissalo, K. Mikhaylov, A. Roivainen and T. Hänninen, "On the coverage of LPWANs: range evaluation and channel attenuation model for LoRa technology," in Proc. Inter. Conf. Intelligent Transport Syst. Telecom., pp. 55-59, Dec. 2015.

[23] N. Abramson, "The ALOHA system-Another alternative for computer communications," Proc. AFIPS Fall Joint Computer Conf., vol. 37, pp. 281-285, Nov. 1970.

[24] J. Haapola et al., "Peer-to-peer energy trading and grid control communications solutions' feasibility assessment based on key performance indicators," IEEE 87th Vehicular Technology Conf. (VTC Spring), pp. 1 - 5, June 2018 